\title{
Non-explosion of solutions to fuzzy stochastic differential equations with non- Lipschitz coefficients
}

\author{
Weiyin Fei, Dandan He, Hongjian Liu \\ School of Mathematics and Physics \\ Anhui Polytechnic University \\ Wuhu 241000, China \\ e-mail: wyfei@ahpu.edu.cn.
}

\begin{abstract}
A class of fuzzy stochastic differential equations (FSDEs) with non-Lipschitzian coefficients is discussed. We first give the preliminaries on the fuzzy stochastic differential equations. Then the non-explosion of solutions to the FSDEs is studied. Finally, the conclusion is given.
\end{abstract}

Keywords- fuzzy stochastic differential equations; nonLipschitzian condition; non-explosion; Bihari's ineqaulity; support function

\section{INTRODUCTION}

For the growing population we have the deterministic Malthus model $d x(t)=a x(t) d t, \quad x(0)=p_{0}$ (see, e.g., Barros et al.[1]).

However, the fuzzy structure can be introduced. We can suppose that the population follows the Malthusian growth, according to the model

$$
d x(t)=a x(t) d t, x(0)=u_{0} \in \mathcal{F}(\mathbf{R}), a \in \mathbf{R} .
$$

Since $u_{0}$ is a fuzzy set in $\mathcal{F}(\mathbf{R})$ which will be defined in the next section, the field $f(u)=a u$ associates fuzzy sets to fuzzy sets. The solutions of this Malthusian equation are discussed in Barros et al. [1]. Also, the topic of fuzzy differential equations has been rapidly growing in recent years (e.g., see Bede et al.[2], Ding et al. [3], Kaleva [12], [13] and Nieto [22]). Motivated by this type of above problems, Romàn-Flores and Rojas-Medar [25] presented some results on the existence of solutions for the fuzzy Cauchy problem under compactness conditions.

In real would, the outcomes of a random experiment often are not exact but are expressed in inexact linguistic variables which change with the time parameter. We can characterize them by the fuzzy stochastic processes.

In fact, fuzzy random variables have been introduced by many authors. We refer to the interesting works of Puri and Ralescu [24] for details, where they make mathematical descriptions for the fuzzy stochastic phenomenon only one time.

Therefore, it is not enough to describe and observe them only one for dynamic fuzzy random phenomena, but it should be done repeatedly and even continuously to describe and observe their evolutional procedures. For this, the theory of the fuzzy stochastic processes is developed (e.g. see Fei [4,5], Fei and Wu [9], Fei et al. [10], Li and Guan [15], Li and Ren [16], Stojaković[27]).

We know that a large class of physically important problem is described by fuzzy random differential systems. Recently, the fuzzy random differential systems are investigated by some researchers, e.g., Fei [8], Kim [14], Malinowski [17-19], Malinowski and Michta [20], and Song et al. [26]. The existence and uniqueness of solution for fuzzy random differential equations with non-Lipschitz coefficients are discussed in Fei [6,7], where the following fuzzy random differential equation is discussed

$$
d X(t)=f(t, X(t)) d t, X(0)=x_{0},
$$

where for each $\omega \in \Omega$,

$$
f(t, x, \omega): \mathbf{R}_{+} \times \mathcal{F}\left(\mathbf{R}^{d}\right) \rightarrow \mathcal{F}\left(\mathbf{R}^{d}\right)
$$

is continuous in the sense of metric $d_{\infty}$.

However, in a real problem, a fuzzy random system is often disturbed by a random noise, such as a Brownian motion. In our system analysis, we are faced with the disturbed term in Eq. (2), where the single valued nose term can be expressed as the stochastic integral of the Brownian motion $W(t)$. Thus we formulate the fuzzy stochastic system as follows

$$
d X(t)=f(t, X(t)) d t+\prec g(t, X(t)) d W(t) \succ, X(0)=x_{0},
$$

where $\prec \cdot \succ$ is the fuzzification of single value which will be explained later. In fact, the properties of solutions to Eq. (3) under some conditions have been investigated in Fei [8], Malinowski [18, 19] and Malinowski and Michta [20].

In this paper, we try to study the behaviour of the solutions to Eq. (3), which are essential in the theory of the fuzzy stochastic dynamical system analysis. In order to prove our claim, the generalized Bihari's lemma is provided, the concept of support function is needed. In the process of the proof of the theorem, the technique to construct a function $G$ is used, which can refer to Fei [6, 7].

The organization of the paper is as follows. In Section 2, we give preliminaries and the lemma.In Section 3, the liftime of the solution to Itô type FSDEs are studied. Moreover, the dependence of the solution to FSDEs on initial values is discussed. In Section 4 we conclude. 


\section{PREliminaries}

Throughout this paper, let $I$ be a finite or infinitely interval and $\left(\Omega, \mathcal{A}, \mathcal{P} ;\left\{\mathcal{A}_{t}\right\}_{t \in I}\right)$ be a complete, filtered probability space where the sub-sigma-field family $\left(\mathcal{A}_{t}, t \in I\right)$ of $\mathcal{A}$ satisfies the usual conditions. Let $\prec \cdot \succ: \mathbf{R}^{d} \rightarrow \mathcal{F}\left(\mathbf{R}^{d}\right)$ denote the embedding of $\mathbf{R}^{d}$, i.e. for $q \in \mathbf{R}^{d}$ we have

Set

$$
\prec q \succ(a)=\left\{\begin{array}{l}
1 \text {, if } a=q, \\
0 \text {, if } a \in \mathrm{R}^{d} \backslash q .
\end{array}\right.
$$

$\mathcal{P}\left(\mathbf{R}^{d}\right) \triangleq\left\{A \subset \mathbf{R}^{d}: A\right.$ is nonempty, compact convex set $\}$. Define

$$
\|A\| \triangleq \sup \{\|x\|: x \in A\} \text { for } A \subset \mathbf{R}^{d} .
$$
$\sigma(A, r) \triangleq \sup \{\langle x, r\rangle: x \in A\}, r \in \mathbf{B}$ is the support function of $A$, where $\mathbf{B}$ is a unit sphere centered at origin. Similar to Fei [5], Fei and Wu [9], we define the operation of addition, minus and "open” in $\mathcal{P}\left(\mathbf{R}^{d}\right)$ as follows:

(i) $A+B=\{x+y: x \in A, y \in B\}$,

(ii) $A \ominus B=\{x: \forall y \in B, \exists z \in A$, s.t. $x+y=z\}$,

(iii) If $\exists C \in \mathcal{P}\left(\mathbf{R}^{d}\right)$, s.t. $A=B+C$, then $A$ is called “open” with respect to (w.r.t.) $B$.

In this paper a fuzzy set $u \in \mathcal{F}\left(\mathbf{R}^{d}\right)$ is a function $u: \mathbf{R}^{d} \rightarrow[0,1]$ for which the $\alpha$-level set $[u]^{\alpha}$ of $u$, defined by $[u]^{\alpha}=\left\{x \in \mathbf{R}^{d}: u(x) \geq \alpha\right\}$ is nonempty, compact and convex subset of $\mathbf{R}^{d}$ for all $\alpha \in(0,1]$. Also $[u]^{0}=\overline{\left\{x \in \mathbf{R}^{d}: u(x)>0\right\}}$ is compact.

A fuzzy random variable is a function

$$
X: \Omega \rightarrow \mathcal{F}\left(\mathbf{R}^{d}\right)
$$

such that

$$
\left\{(\omega, x): x \in[X(\omega)]^{\alpha}\right\} \in \mathcal{A} \times \mathcal{B}\left(\mathbf{R}^{d}\right), \forall \alpha \in(0,1],
$$
where $\mathcal{B}\left(\mathbf{R}^{d}\right)$ denotes the Borel subsets of $\mathbf{R}^{d}$. $\|X\| \triangleq\left\|[X]^{0}\right\|=\sup _{x \in[X]^{0}}\|x\|$.

If $H$ is Hausdorff metric defined on $\mathcal{P}\left(\mathbf{R}^{d}\right)$, $H(A, B) \triangleq \max \left\{H^{+}(A, B), H^{-}(B, A)\right\}, A, B \in \mathcal{P}\left(\mathbf{R}^{d}\right)$, where

$$
H^{+}(A, B) \triangleq \sup _{x \in A} \inf _{y \in B}\|x-y\|, H^{-}(A, B)=H^{+}(B, A) \text {, }
$$

then $\left(\mathcal{P}\left(\mathbf{R}^{d}\right), H\right)$ is a complete metric space.

For two fuzzy sets $u, v \in \mathcal{F}\left(\mathbf{R}^{d}\right)$ we can define a distance $d_{\infty}: \mathcal{F}\left(\mathbf{R}^{d}\right) \times \mathcal{F}\left(\mathbf{R}^{d}\right) \rightarrow \mathbf{R}_{+}$by

$$
d_{\infty}(u, v)=\sup _{0 \leq \alpha \leq 1} H\left([u]^{\alpha},[v]^{\alpha}\right) .
$$

Two fuzzy random variables $X, Y$ with values in $\mathcal{F}\left(\mathbf{R}^{d}\right)$ are considered to be identical if $d_{\infty}(X, Y)=0$ a.s..

We define the operation on $\mathcal{F}\left(\mathbf{R}^{d}\right)$ as follows. For two fuzzy sets $u, v \in \mathcal{F}\left(\mathbf{R}^{d}\right)$, if there exists such a $w \in \mathcal{F}\left(\mathbf{R}^{d}\right)$ that $u=v+w$, then $w$ is the Hukuhara difference of $u$ and $v$ denoted by $w=u \ominus v$. We note that $u+v=\prec 0 \succ$ implies that $u=-v$. However, $u \ominus u \neq \prec 0 \succ$. Indeed, take $u=\chi_{[0.1]}$.

It is well known that

$d_{\infty}(u+w, v+w)=d_{\infty}(u, v), d_{\infty}(\lambda u, \lambda v)=|\lambda| d_{\infty}(u, v)$ for all $u, v, w \in \mathcal{F}_{w c}(X), \lambda \in \mathbf{R}$.

Denote $\sigma(u, \alpha, r) \equiv \sigma_{u}(\alpha, r) \triangleq \sigma\left([u]^{\alpha}, r\right), \forall r \in \mathbf{B}, \alpha \in[0,1], u \in \mathcal{F}\left(\mathbf{R}^{d}\right)$.

The support function $\sigma_{u}$ satisfies following properties:

(i) $\sigma_{u}$ is uniformly bounded on $[0,1] \times \mathbf{B}$,

$$
\left|\sigma_{u}\left(\alpha, x^{*}\right)\right| \leq \sup _{x \in[u]^{0}}\|x\|=\|u\| .
$$

(ii) $\sigma_{u}(\cdot, r)$ is nonincreasing and left-continuous, rightcontinuous at 0 in $\alpha$ for each $r \in \mathbf{B}$.

(iii) $\sigma_{u}(\alpha, \cdot)$ is Lipschitz continuous in $r$ uniformly in $\alpha \in[0,1]$ :

$$
\left|\sigma_{u}(\alpha, r)-\sigma_{u}\left(\alpha, r^{*}\right)\right| \leq\|u\| \cdot\left|r-r^{*}\right| \text {. }
$$

(iv) for all $\alpha \in[0,1]$ and $u, v \in \mathcal{F}\left(\mathbf{R}^{d}\right)$,

or

$$
H\left([u]^{\alpha},[v]^{\alpha}\right)=\sup _{r \in \mathbf{B}}\left|\sigma_{u}(\alpha, r)-\sigma_{v}(\alpha, r)\right|,
$$

$$
d_{\infty}(u, v)=\sup _{(\alpha, r) \in[0,1] \times \mathbf{B}}\left|\sigma_{u}(\alpha, r)-\sigma_{v}(\alpha, r)\right| .
$$

In what follows, we call $X: I \times \Omega \rightarrow \mathcal{F}\left(\mathbf{R}^{d}\right)$ a fuzzy stochastic process, if for every $t \in I$ a mapping $X(t, \cdot)=X(t): \Omega \rightarrow \mathcal{F}\left(\mathbf{R}^{d}\right)$ is a fuzzy random variable. If $X: I \times \Omega \rightarrow \mathcal{F}\left(\mathbf{R}^{d}\right) \quad$ is $\left\{A_{t}\right\}_{t \in I}$-adapted and measurable, then it will be called nonanticipating.

Equivalently, the process $X$ is nonanticipating if and only if for every $\alpha \in[0,1]$ the multifunction $[X]^{\alpha}$ is measurable with respect to the $\sigma$-algebra $\mathcal{N}$, which is defined as follows

$$
\mathcal{N}:=\left\{A \in \mathcal{B}(I) \otimes \mathcal{A}: A^{t} \in \mathcal{A}_{t} \text { for every } t \in I\right\},
$$
where $A^{t}=\{\omega:(t, \omega) \in A\}$ for $t \in I$. 
Let $p \geq 1$ and $L^{p}\left(I \times \Omega, \mathcal{N} ; \mathbf{R}^{d}\right)$ denote the set of all nonanticipating $\mathbf{R}^{d}$-valued stochastic processes $\{h(t)\}_{t \in I}$ such that $E\left(\int_{0}^{T}\|h(s)\|^{p} d s\right)<\infty$, where $\|\cdot\|$ denotes the norm of a vector or matrix. A fuzzy stochastic process $X$ is called $L^{p}$-integrably bounded, if there exists a real-valued stochastic process $h \in L^{p}\left(I \times \Omega, \mathcal{N} ; \mathbf{R}_{+}\right)$such that

$$
\left\|[X(t, \omega)]^{0}\right\| \leq h(t, \omega) \text { for a.a. }(t, \omega) \in I \times \Omega \text {. }
$$

It is easy to see that if $X: \Omega \rightarrow \mathbf{R}^{d}$ is an $\mathbf{R}^{d}$-valued random variable defined on a probability space $(\Omega, \mathcal{A}, P)$, then $\prec X \succ: \Omega \rightarrow \mathcal{F}\left(\mathbf{R}^{d}\right)$ is a fuzzy random variable.

We easily know that $\sigma(X(t), \alpha, r)$ is continuous at $t$ for $\forall \alpha \in[0,1], r \in \mathbf{B}$ if $X(t)$ is $d_{\infty}$-continuous. $X(t)$ is called a $d_{\infty}$-continuous function on $I$ if $X(t)$ is a $d_{\infty}$-continuous function at any $t \in I$. In order to introduce the FSDEs in next section, we give the following definition of the Riemann integral.

Definition 1 Let $X(t)$ be a fuzzy stochastic process defined on $[a, b]$. For each finite partition $\Delta_{n}$ of $[a, b]: \Delta_{n}: a=t_{0}<t_{1}<\cdots<t_{n}=b$, and for arbitrary points $t_{i}^{\prime}, t_{i-1} \leq t_{i}^{\prime} \leq t_{i}, i=1,2, \cdots, n$,

let $S_{n}=\sum_{i=1}^{n} \Delta t_{i} X\left(t_{i}^{\prime}\right)$ and $|\Delta|=\max _{1 \leq i \leq n} \Delta t_{i}$, where $\Delta t_{i}=t_{i}-t_{i-1}$. Then the $d_{\infty}$ Riemann integral-integral) of $X(t)$ on $[a, b]$ is defined by

$$
\int_{a}^{b} X(t) d t \triangleq d_{\infty}-\lim _{\left|\Delta_{n}\right| \rightarrow 0} S_{n}
$$

provided this limit exists and it is independent of the partition as well as the selected points $t_{i}^{\prime}$.

In what follows, we provide the generalize Bihari's inequality (cf., Fei [17] and Mao [21]) which plays an important role in the following section.

Lemma 1 (Generalized Bihari's inequality)\} Let $u$ be Borel measurable, bounded, nonnegative and left limit function on $\left[0, T_{1}\right]$ and $c>0$. Let $K: \mathbf{R}_{+} \rightarrow \mathbf{R}_{+}$be a continuous nondecreasing function such that $K(t)>0$ for all $t>0$.

(i) If $\mu(t)$ is a continuous nonnegative nondecreasing function on $\left[0, T_{1}\right]$, then the inequality

$$
u(t) \leq c+\int_{0}^{t} K(u(s-)) d \mu(s), \forall t \in\left[0, T_{1}\right]
$$

implies that

$$
u(t) \leq G^{-1}(G(c)+\mu(t))
$$

for all $t \in\left[0, T_{1}\right]$ such that

where

$$
G(c)+\mu(t) \in \operatorname{Dom}\left(G^{-1}\right),
$$

$$
G(q)=\int_{a}^{q} \frac{1}{K(v)} d v, q>0,
$$

$G^{-1}$ is the inverse function of $G$ and $a \in\left[0, T_{1}\right]$.

(ii) If $v(t)$ is a continuous nonpositive nonincreasing function on $\left[0, T_{1}\right]$, then the inequality

implies that

$$
u(t) \geq c+\int_{0}^{t} K(u(s-)) d v(s), \forall t \in\left[0, T_{1}\right]
$$

$$
u(t) \geq G^{-1}(G(c)+v(t))
$$

for all $t \in\left[0, T_{1}\right]$ such that

$$
G(c)+v(t) \in \operatorname{Dom}\left(G^{-1}\right) .
$$

\section{FUZZY STOCHASTIC DIFFERENTIAL EQUATIONS}

In this section, we consider the following fuzzy stochastic differential equation (in the integral form)

$$
X(t)=x_{0}+\int_{0}^{t} f(X(s)) d s+\prec \int_{0}^{t} g(X(s)) d W(s) \succ, \quad \text { (4) }
$$

where $f$ takes values in $C\left(\mathcal{F}\left(\mathbf{R}^{d}\right), \mathcal{F}\left(\mathbf{R}^{d}\right)\right), \quad g$ in $C\left(\mathcal{F}\left(\mathbf{R}^{d}\right), \mathbf{R}^{d} \times \mathbf{R}^{m}\right), \quad W=(W(t), t \geq 0)$ is an $m-$ dimensional Brownian motion, and $x_{0} \in \mathcal{F}\left(\mathbf{R}^{d}\right)$ is a fuzzy random variable. Here, $C\left(\mathcal{F}\left(\mathbf{R}^{d}\right), \mathbf{R}^{d} \times \mathbf{R}^{m}\right)$ stands for the family of the continuous functions from the fuzzy space $\mathcal{F}\left(\mathbf{R}^{d}\right)$ to the space $\mathbf{R}^{d} \times \mathbf{R}^{m}$. In Eq. (4), the integral $\int_{0}^{t} f(X(s)) d s$ is defined in Definition 1, and the integral $\int_{0}^{t} g(X(s)) d W(s)$ is a general Itô type stochastic one, whose definition can refer to Øksendal [23].

From the continuouty of $f$, we can know that $\sigma(f(\cdot), \alpha, r)$ takes values in $C\left(\mathcal{F}\left(\mathbf{R}^{d}\right), \mathbf{R}\right)$ which is the family of the continuous functions from the fuzzy space $\mathcal{F}\left(\mathbf{R}^{d}\right)$ to the space $\mathbf{R}$. Similar to the discussion of Ikeda and Watanabe [11] and Stroock and Varadhan [28], it is known that the following stochastic differential equation

$$
\begin{aligned}
d \sigma(X(t), \alpha, r)= & \sigma(f(X(t)), \alpha, r) d t+r^{\top} g(X(t)) d W(t), \\
& \sigma(X(0), \alpha, r)=\sigma\left(x_{0}, \alpha, r\right)
\end{aligned}
$$

has a solution up to a lifetime $\zeta(\alpha, r)$ which depends on $(\alpha, r) \in[0,1] \times \mathbf{B}$. Set

$$
\zeta=\inf _{\alpha \times r \in[0,1] \times \mathbf{B}} \zeta(\alpha, r)
$$

we call $\zeta$ the lifetime of solution to Eq. (4). Obviously, $\|X(\zeta)\|=+\infty$. 
If Eq. (5) has the pathwise uniqueness, then we show the existence and uniqueness of the solution to the FSDE(4). So the study of pathwise uniqueness is of great interest. It is a classical result that under the Lipschitz coefficients, the pathwise uniqueness holds and the solution of Eq. (5) can be constructed by using iteration; moreover the solution depends on the initial values continuously. In what follows, we shall deal with a class of non-Lipschitzian FSDEs. Presently, the existence and uniqueness of solutions to Eq. (4) or Eq. (5) can be proven similar to the one in Fei [8]. The main tool of these studies is the generalized Bihari's inequality. Our idea is to derive an inequality so that the generalized Bihari's inequality can be applied. For a study of a class of fuzzy random differential equation under nonLipschitzian coefficients without the Brownian noise, we refer to Fei [6]. The following result weakens the linear growth condition of non-explosion.

Theorem 1 Let $\rho: \mathbf{R}_{+} \rightarrow[1,+\infty)$ be a continuous function such that

(i) $s \rho(s)$ is nondecreasing and concave;

(ii) $\int_{0}^{\infty} \frac{d s}{s \rho(s)+1}=+\infty$.

Assume that for some constant $C>0$,

$$
\begin{aligned}
& \|f(x)\| \leq C\left(\|x\| \rho\left(\|x\|^{2}\right)+1\right), \\
& \|g(x)\|^{2} \leq C\left(\|x\|^{2} \rho\left(\|x\|^{2}\right)+1\right), x \in \mathcal{F}\left(\mathbf{R}^{d}\right) .
\end{aligned}
$$

If $E\left\|x_{0}\right\|<+\infty$, then the lifetime of the solution $X\left(t, x_{0}\right)$ to Eq. (4) is infinite: $\zeta=+\infty$.

Proof. Let

$$
\lim _{E\left\|x_{0}\right\| \rightarrow+\infty} E\left\|X\left(t, x_{0}\right)\right\|=+\infty \text {. }
$$

$$
\xi^{\alpha, r}(t)=|\sigma(X(t), \alpha, r)|^{2}, \forall(\alpha, r) \in[0,1] \times \mathbf{B},
$$

where $X(t)$ is a solution to Eq. (4). Hence we have

$$
\begin{aligned}
& d \xi^{\alpha, r}(t)= \\
& {\left[2 \sigma(X(t), \alpha, r) \sigma(f(X(t)), \alpha, r)+\left\|r^{\top} g(X(t))\right\|^{2}\right] d t} \\
& +2 \sigma(X(t), \alpha, r) r^{\top} g(X(t)) d W(t) .
\end{aligned}
$$

Since $r \in \mathbf{B}$, we get $\left\|r^{\top} g(X(s))\right\|^{2} \leq\|g(X(s))\|^{2}$.

Set $\xi(t)=\|X(t)\|^{2}$, it follows that from (6) and the property (i) of the support function $\sigma(\cdot)$

$$
\begin{aligned}
& E \xi^{\alpha, r}(t)=\xi^{\alpha, r}(0) \\
& \quad+2 E \int_{0}^{t}(\sigma(X(s), \alpha, r) \sigma(f(X(s)), \alpha, r) \\
& \left.\quad+\left\|r^{\top} g(X(s))\right\|^{2}\right) d s \\
& \leq E\left\|x_{0}\right\|^{2}+2 C \int_{0}^{t} E(2 \xi(s) \rho(\xi(s))+\sqrt{\xi(s)}+1) d s \\
& \leq E\left\|x_{0}\right\|^{2}+2 C \int_{0}^{t}(2 E \xi(s) \rho(E \xi(s))+\sqrt{E \xi(s)}+1) d s,
\end{aligned}
$$

where we have utilized the concavity of the functions $v \rho(v)$ and $\sqrt{v}$.
Denote $\eta(t)=E \xi(t)$. Noticing $\rho(\eta) \geq 1$, we get

$$
\frac{\eta \rho(\eta)+\sqrt{\eta}}{\eta \rho(\eta)+1} \leq 1+\frac{\sqrt{\eta}}{\eta \rho(\eta)+1} \leq 1+\frac{\sqrt{\eta}}{\eta+1} \leq 1+\frac{1}{2}<2,
$$

where $\varphi(\eta)=\frac{\sqrt{\eta}}{1+\eta}$ takes the maximum at 1 on the interval $(0, \infty)$.

It is easy to see that

which deduce that

$$
\sup _{\eta \geq 0} \frac{\eta \rho(\eta)+\sqrt{\eta}}{\eta \rho(\eta)+1} \leq 2
$$

$$
\frac{2 \eta \rho(\eta)+\sqrt{\eta}}{\eta \rho(\eta)+1} \leq \frac{2(\eta \rho(\eta)+\sqrt{\eta})}{\eta \rho(\eta)+1}+\frac{1}{\eta \rho(\eta)+1} \leq 5 \text {. }
$$

Thus we have

$$
2 \eta \rho(\eta)+\sqrt{\eta}+1 \leq 6(\eta \rho(\eta)+1)
$$

In virtue of (7), we have

$$
\eta(t) \leq E\left\|x_{0}\right\|^{2}+12 C \int_{0}^{t}(\eta(s) \rho(\eta(s))+1) d s .
$$

Set

$$
G(u)=\int_{1}^{u} \frac{d s}{s \rho(s)+1}, u>0
$$

By the condition (ii) it is easy to show that $G(u)$ is strictly increasing,

$$
G(u) \rightarrow+\infty \text { as } u \rightarrow+\infty
$$

and $G^{-1}(u) \rightarrow+\infty$ as $u \rightarrow+\infty$.

From the generalized Bihari's inequality (Lemma 1(i)), and $E \mid\left\|x_{0}\right\|<+\infty$ we obtain

$$
\eta(t) \leq G^{-1}\left(G\left(E\left\|x_{0}\right\|^{2}\right)+12 C t\right)<+\infty, \forall t \geq 0,
$$

which proves that $\zeta=+\infty$.

On the other hand, from (6) and the property (i) of the support function $\sigma(\cdot)$ we have

$$
\begin{aligned}
& E \xi^{\alpha, r}(t)=\xi^{\alpha, r}(0) \\
& \quad+2 E \int_{0}^{t}(\sigma(X(s), \alpha, r) \sigma(f(X(s)), \alpha, r) \\
& \left.\quad+\left\|r^{\top} g(X(s))\right\|^{2}\right) d s \\
& \geq E\left\|x_{0}\right\|^{2}-2 C \int_{0}^{t} E(\xi(s) \rho(\xi(s))+\sqrt{\xi(s)}) d s \\
& \geq E\left\|x_{0}\right\|^{2}-2 C \int_{0}^{t}(E \xi(s) \rho(E \xi(s))+\sqrt{E \xi(s)}) d s .
\end{aligned}
$$

Due to the inequality (8), we have

$$
\eta(t) \geq E\left\|x_{0}\right\|^{2}-4 C \int_{0}^{t}(\eta(s) \rho(\eta(s))+1) d s .
$$

By Lemma 1(ii), we get

$E\left\|X\left(t, x_{0}\right)\right\|^{2}=\eta(t) \geq G^{-1}\left(G\left(E\left\|x_{0}\right\|^{2}\right)-4 C t\right), \forall t \geq 0$, which shows that $\lim _{E\left\|x_{0}\right\| \rightarrow+\infty} E\left\|X\left(t, x_{0}\right)\right\|=+\infty$ by the 
property of the function $G(\cdot)$ in (10). Thus, the proof of theorem is complete.

\section{ConClusion}

In real world, we are often faced with random experiments whose outcomes are not exact but are expressed in inexact linguistic variables which change with the time parameter. The fuzzy stochastic differential systems can be used to describe a large class of physically important problem. On the other hand, the fuzzy stochastic systems are often disturbed by a Borwnian noise. In this paper, we investigate the behavior of solutions to the FSDEs driven by a Wiener process under the non-Lipschitzian coefficients.

The lifetime of solutions to the FSDEs of Itô type is characterized through using the support function and Bihari's inequality. Furthermore, the dependence of solutions to FSDEs with respect to the initial data is analysed. The other related properties of the solutions to FSDEs driven by a Wiener process will be discussed in future.

\section{ACKNOWLEDGMENT}

This paper is supported by the National Natural Science Foundation of China (71171003; 71271003), Anhui Natural Science Foundation (090416225) and Anhui Natural Science Foundation of Universities (KJ2010A037).

\section{REFERENCES}

[1] L.C. Barros, R.C. Bassanezi, P.A. Tonelli, Fuzzy modelling in population dynamics, Ecological Modeling 128 (2000) 27-33.

[2] B. Bede, I.J. Rudas, A.L. Bencsik, First order linear fuzzy differential equations under generalized differentiability, Inform. Sci. 177 (2007) 1648-1662.

[3] Z. Ding, M. Ma, A. Kandel, Existence of the solutions of fuzzy differential equations with parameters, Inform. Sci. 99 (1999) 205217.

[4] W.Y. Fei, Regularity and stopping theorem for fuzzy artingales with continuous parameters, Inform. Sci. 169 (2005) 175-187.

[5] W.Y. Fei, On the theory of (dual) projection for fuzzy stochastic processes, Stoch. Anal. Appl. 23 (2005) 449-474.

[6] W.Y. Fei, Existence and uniqueness of solution for fuzzy random differential equations with non-Lipschitz coefficients, Inform. Sci. 177 (2007) 4329-4337.

[7] W.Y. Fei. A generalization of Bihari's inequality and fuzzy random differential equations with non-Lipschitz coefficients. International Journal of Uncertainty, Fuzziness and Knowledge-Based Systems 15(4) (2007) 425-439

[8] W.Y. Fei. Existence and uniqueness for solutions to fuzzy stochastic differential equations driven by local martingales under the nonLipschitzian condition. Nonlinear Analysis: TMA 76 (2013) 202-214.

[9] W.Y. Fei, R.Q. Wu, Doob's decomposition theorem for fuzzy (super) submartingales, Stoch. Anal. Appl. 22 (2004) 627-645.

[10] W.Y. Fei, R.Q. Wu, S.H. Shao, Doob's stopping theorem for fuzzy (super, sub) martingales with discrete times, Fuzzy Sets and Systems 135 (2003) 377-390.

[11] N. Ikeda, S. Watanabe, Stochastic Differential Equations and Diffusion Processes, North-Holland, Amsterdam, 1981.

[12] O. Kaleva, The Cauchy problem for fuzzy differential equations, Fuzzy Sets and Systems 35 (1990) 389-396.
[13] O. Kaleva, A note on fuzzy differential equations, Nonlinear Analysis 64 (2006) 895-900.

[14] J.H. Kim, On fuzzy stochastic differential equations, J. Korean Math. Soc. 42 (2005) 153-169.

[15] S. Li, L. Guan, Fuzzy set-valued Gaussian processes and Brownian motions, Inform. Sci. 177 (2007) 3251-3259.

[16] S. Li, A. Ren, Representation theorems, set-valued and fuzzy setvalued Ito integral, Fuzzy Sets and Systems 158 (2007) 949-962.

[17] M.T. Malinowski, Random fuzzy differential equations under generalized Lipschitz condition, Nonlinear Anal. Real World Appl. 13 (2012) 860-881.

[18] M.T. Malinowski, Strong solutions to stochastic fuzzy differential equations of Itô type, Math. Comput. Modelling 55 (2012) 918-928.

[19] M.T. Malinowski, Itô type stochastic fuzzy differential equations with delay, Systems \& Control Letters 61 (2012) 692-701.

[20] M.T. Malinowski, M. Michta, Stochastic fuzzy differential equations with an application, Kybernetika 47 (2011) 123-143.

[21] X.R. Mao, Exponential Stability of Stochastic Differential Equation, Marcel Dekker, New York, 1994.

[22] J.J. Nieto, The Cauchy problem for continuous fuzzy differential equations, Fuzzy Sets and Systems 102 (1999) 259-262.

[23] B. Øksendal, Stochastic Differential Equations: An Introduction with Applications, 6th Ed.,Springer-Verlag, Berlin, 2005.

[24] M.L. Puri, D.A. Ralescu, Fuzzy random variables, J. Math. Anal. Appl. 114 (1986) 406-442.

[25] H. Romàn-Flores, M. Rojas-Medar, Embedding of level-continuous fuzzy sets on Banach spaces, Inform. Sci. 144 (2002) 227-247.

[26] S.J. Song, C. Wu, X.P. Xue, Existence and uniqueness of Cauchy problem for fuzzy differential equations under dissipative conditions, Comput. Math. Appl. 51 (2006) 1483-1492.

[27] M. Stojaković, Fuzzy martingales-a simple form of fuzzy processes, Stoch. Anal. Appl. 14(3) (1996) 355-367.

[28] D.W. Stroock, S.R.S. Varadhan, Multidimensional Diffusion Processes, Springer-Verlag, Berlin, 1979. 